\title{
26. MASS-ACCUMULATION RATES OF THE NON-AUTHIGENIC INORGANIC CRYSTALLINE (EOLIAN) COMPONENT OF DEEP-SEA SEDIMENTS FROM HESS RISE, DEEP SEA DRILLING PROJECT SITES 464, 465, AND $466^{1}$
}

\author{
David K. Rea and Eileen C. Harrsch, Oceanography Program, Department of Atmospheric and Oceanic Science,
} The University of Michigan, Ann Arbor, Michigan

\begin{abstract}
The mass-accumulation rate (MAR) of the non-authigenic, inorganic, crystalline component of deep-sea sediments from the Pacific aseismic rises apparently reflects influx of eolian sediment. The eolian sediment usually is dominated by volcanic material, except during glacial times. Sediments from Hess Rise provide a discontinuous record of eolian MARs. During Albian to Cenomanian time, the influx of volcanic material was fairly high $\left(0.35-0.6 \mathrm{~g} / \mathrm{cm}^{2} / 10^{3} \mathrm{yr}\right)$, recording the latest stages of the Albian volcanism that formed Hess Rise. From the Campanian through the Paleocene, influx of eolian sediment was low, averaging $0.03 \mathrm{~g} / \mathrm{cm}^{2} / 10^{3} \mathrm{yr}$. None of the four Hess Rise drill sites show evidence of the Late Cretaceous volcanic episode recorded at many sites now in the equatorial to subtropical Pacific. Pliocene to Pleistocene samples record a peak in volcanic influx about 4 to $5 \mathrm{~m}$.y. ago, which has been well documented elsewhere. The several-fold increase in eolian accumulation rates elsewhere which are correlated with the onset of severe northernhemisphere glaciation 2.5 m.y. ago is not obvious in the Hess Rise data.
\end{abstract}

\section{INTRODUCTION}

\section{Background and Objectives}

DSDP Leg 62 was planned to investigate the paleoenvironment of the North Pacific Ocean. Hess Rise was chosen as the location for three drill sites, because its crestal parts lie near or above the calcite compensation depth, thus greatly increasing the chance of encountering well-preserved sections of calcareous biogenic sediment. It was hoped that sediments from Hess Rise and the Mid-Pacific Mountains would provide the basis for a detailed reconstruction of the Pacific oceanic environment from the present back to middle Cretaceous time.

The North Pacific aseismic rises (Hess, Shatsky, and the plateau level of the Mid-Pacific Mountains) are also among the best places for studying the history of eolian deposition. These areas probably have always been above the region of nepheloid-layer transport of finegrained sediments below about 4500 meters. Second, these sites are far from continental margins, and thus beyond the realm of hemipelagic deposition. Furthermore, the rises have been south of the polar front (subpolar convergence) during Pleistocene nonglacial and glacial intervals (CLIMAP, 1976), and thus are not likely to have been subject to deposition by ice-rafting.

If these three sediment sources can be discounted, then the most significant remaining non-authigenic, inorganic, crystalline materials are the dust grains carried by the winds. Eolian dust may come from distant continents or local volcanoes, and the mass-accumulation rates presented below record changes in both supply rate and transport efficiency; we are unable to determine which factor is dominant from accumulation-rate data alone. The mineralogy of the Hess Rise sediments

\footnotetext{
${ }^{1}$ Initial Reports of the Deep Sea Drilling Project, Volume 62.
}

(Vallier and Jefferson, this volume) suggests that most of the presumably eolian component is of volcanic origin.

\section{Geologic Setting}

Hess Rise is a triangular elevation bounded by the Mendocino Fracture Zone on the south, the Emperor Trough on the northeast, and the Emperor Seamounts to the west (Fig. 1). Site 464 is on the deeper, northern extension of the rise $\left(39^{\circ} 51.6^{\prime} \mathrm{N}, 173^{\circ} 53.3^{\prime} \mathrm{E} ; 4637 \mathrm{~m}\right.$ deep). Sites $465\left(33^{\circ} 49.2^{\prime} \mathrm{N}, 178^{\circ} 55.1^{\prime} \mathrm{E} ; 2161 \mathrm{~m}\right.$ deep) and $466\left(34^{\circ} 11.5^{\prime} \mathrm{N}, 179^{\circ} 15.3^{\prime} \mathrm{E} ; 2665 \mathrm{~m}\right.$ deep) are on the higher, south-central portion of the rise near Mellish Bank.

The sections cored at these three sites are illustrated in Figure 2. At Site 464, Sub-unit IA is 18.8 meters of clayey radiolarian ooze of Pleistocene to early Pliocene age; Sub-unit IB is $\mathbf{1 7 . 3}$ meters of lower Pliocene and upper Miocene siliceous clay that contains some volcanic glass. Below, there is a rather abrupt change to the underlying brown clays of Unit II. These clays are 52.9 meters thick and range in age from early Miocene to late Cretaceous (Doyle and Riedel, this volume). Unit III consists of 218.6 meters of red-brown chert and nannofossil limestone of Albian and Cenomanian age. Because the recovery from this unit was essentially all chert pieces, there were no subsamples taken for our analysis. Basalt comprises the 0.16 meters of Unit IV.

Drilling in two holes at Site 465 encountered three lithologic units (Fig. 2). Unit I is $276 \mathrm{~m}$ of nannofossil and foraminifer-nannofossil ooze of Pleistocene to Cenomanian age. Lacunae representing Pliocene to Paleocene time, late Campanian to Santonian time, and Santonian to early Cenomanian time occur within Unit I. The Paleocene to upper Campanian section is the best-preserved record of the Cretaceous/Tertiary boundary recovered to date from the Pacific Ocean (Site 465 


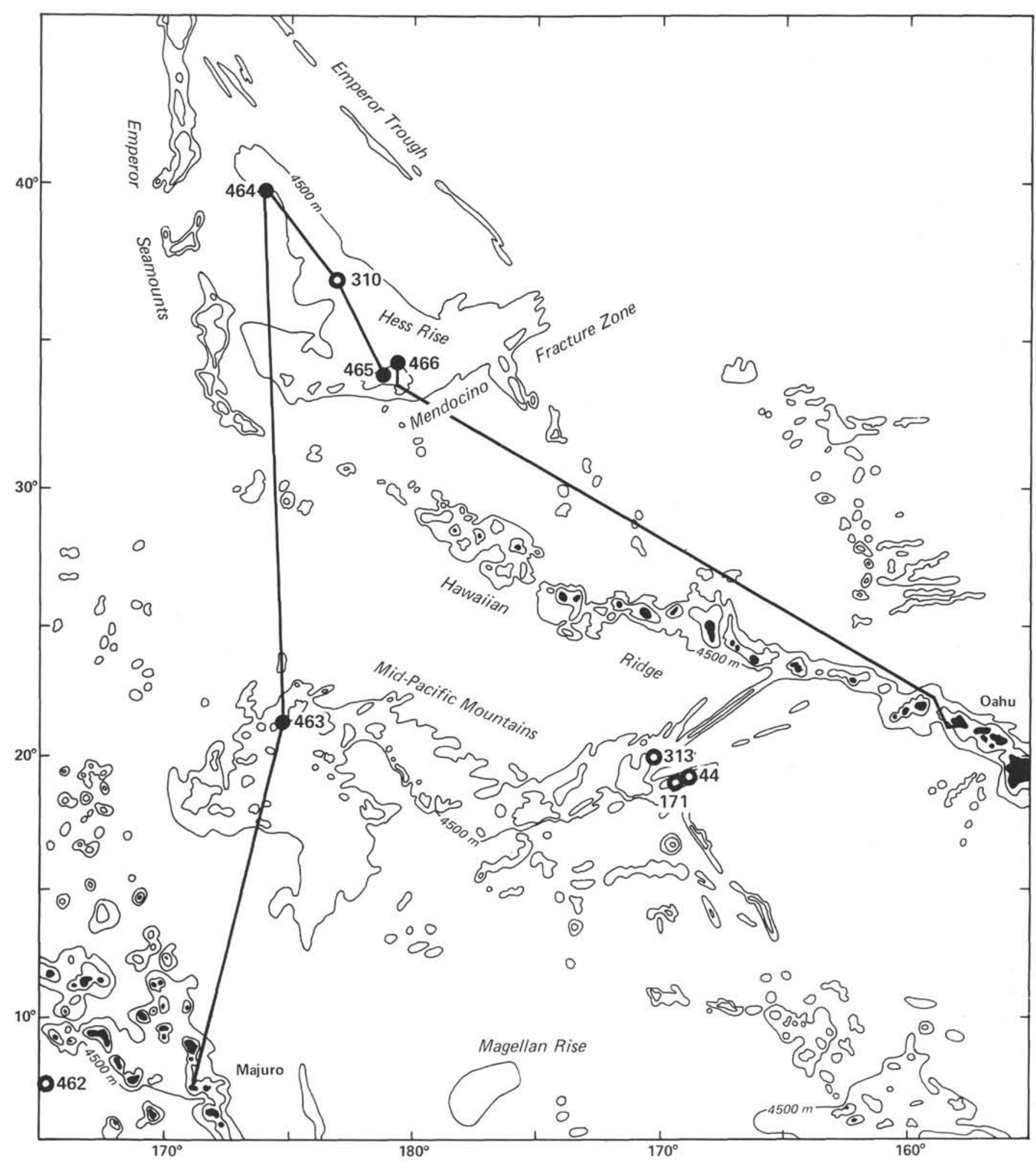

Figure 1. Track line of the Glomar Challenger during DSDP Leg 62, showing the location of the Hess Rise drill sites.

report, this volume). Below the oozes at Site 465 is 135.7 meters of laminated limestone of early Cenomanian to late Albian age which constitutes Unit II. Unit III is 64.3 meters of trachyte.

Site 466 was drilled about $50 \mathrm{~km}$ northeast of 465 , and a similar stratigraphic section was found (Fig. 2).
Sub-unit IA is 84 meters of Pleistocene to upper Eocene nannofossil ooze, Sub-unit IB is 161.5 meters of middle Eocene to Turonian cherty nannofossil ooze; this subunit contains rounded pebbles of alkali basalt and of hematite in the Campanian section (Site 466 report, this volume). Lacunae separate early Pliocene from late 

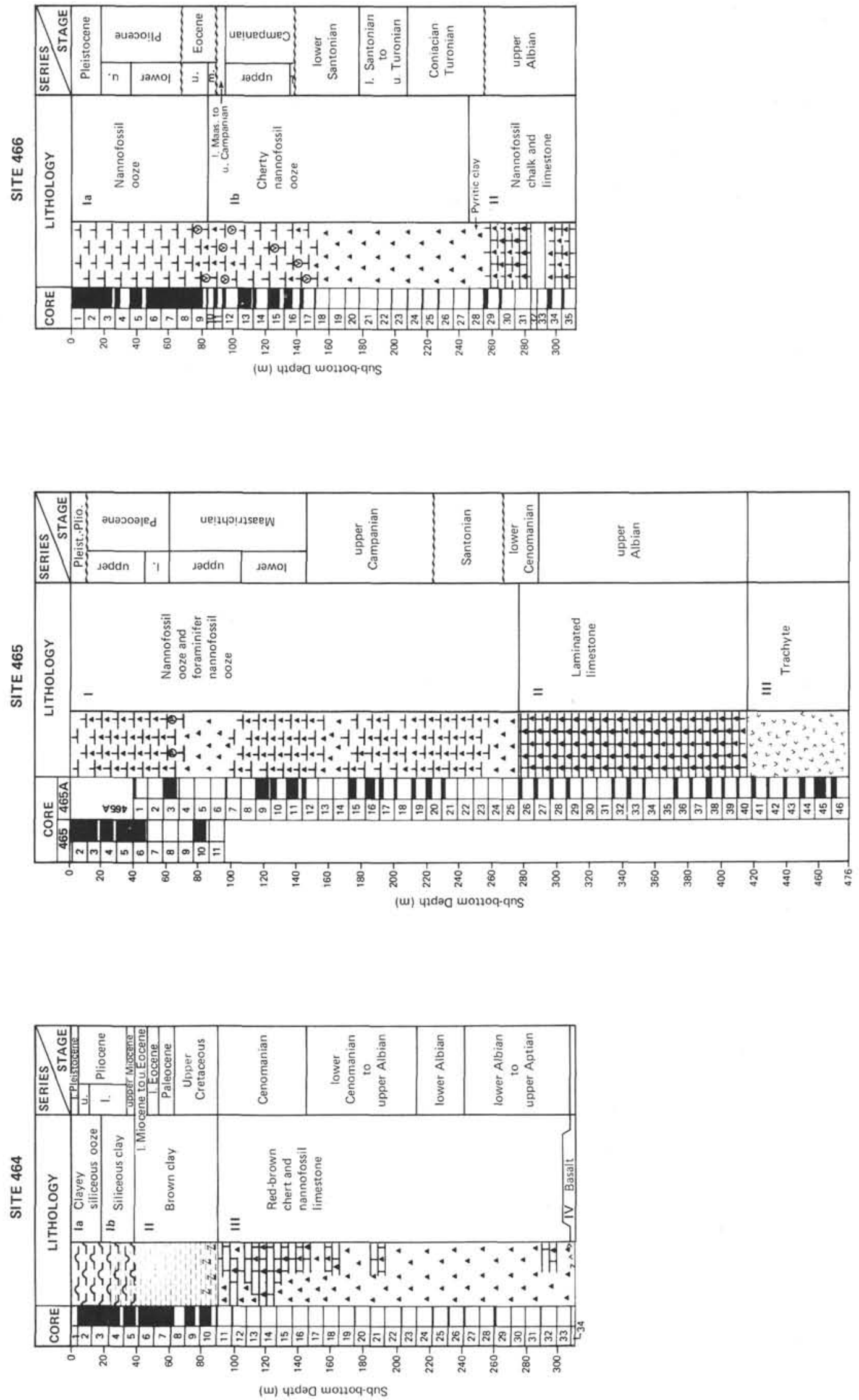
Eocene sediments and middle Eocene from early Maastrichtian sediments. Unit II is composed of 66.5 meters of Turonian to upper Albian olive-gray nannofossil chalk and limestone, containing pyritic clay in the upper part. Drilling at Site 466 did not penetrate volcanic basement.

\section{METHODS}

There are two parameters which describe the rate of deposition of sediment: one, the linear sedimentation rate (LSR), or the length per unit time, undergoes an apparent reduction in value (van Andel et al., 1975 ) as the weight of the overlying sediment compacts the sediment and reduces its porosity; the second parameter, a measure of the drymass flux called the mass-accumulation rate (MAR), eliminates the compaction problem and allows comparison of samples from different depths. To determine the MARs in units of $\mathrm{g} / \mathrm{cm}^{2} / 10^{3} \mathrm{yr}$, we first determined the dry weight of the sediment per unit wet volume. The product of this value, the dry-bulk density, and the LSR yields the mass-accumulation rate. To determine the inorganic MAR, we calculated the product of the weight percent of the non-authigenic, inorganic, crystalline component and the MAR of the sedimentary section.

The non-authigenic, inorganic, crystalline component was isolated by extracting the calcareous, opaline, and oxide-hydroxide portions of the sediment. Details of the laboratory methods are given by Rea and Janecek (this volume).

Tables 1,2 , and 3 are a summary of the dry-bulk densities, porosities, MARs, LSRs, and inorganic MARs at each Hess Rise site. Figures 3 to 6 are plots of the inorganic MARs versus age.

Values that seemed to indicate an important change in MAR were rerun, as were widely discrepant points. We achieved agreement within $5 \%$ of the originally determined value with each replicate, an indication of the precision of our procedures.

\section{MASS-ACCUMULATION RATES}

\section{Site 464}

The LSR determined for the Pliocene siliceous clays of Unit I is $11.3 \mathrm{~m} / \mathrm{m}$.y. (site 464 report, this volume). For our calculations, we have assumed that this rate is valid down to the top of the brown clays of Unit II. The age span of the brown clays is broad and gives a LSR of less than $1 \mathrm{~m} / \mathrm{m}$.y. for Unit II. This section is almost certainly characterized by discontinuous sedimentation (Doyle and Riedel, this volume), so we have not included it in our calculations of the inorganic MAR.
During the Pliocene, the MAR of the non-authigenic, inorganic, crystalline component of sediments at Site 464 was moderately high, fluctuating between 0.2 and $0.4 \mathrm{~g} / \mathrm{cm}^{2} / 10^{3} \mathrm{yr}$ (Table 1; Figs. 3 and 6 ).

\section{Site $\mathbf{4 6 5}$}

At Site 465 , we were able to calculate MARs of the eolian component for materials of Pleistocene to Pliocene, Paleocene to Maastrichtian, and Cenomanian to Albian age (Table 2; Fig. 4). The first two cores at this location were somewhat disturbed and gave evidence of Paleogene to Neogene reworking (Site 465 report, this volume).

Linear sedimentation rates are unusually low for the three samples of youngest nannofossil ooze, about 1 $\mathrm{m} / \mathrm{m}$.y., and ages of these sediments are not well determined. We suspect that a significant part of the PlioPleistocene section has been lost (or was never deposited). Sedimentation rates for the entire section are given in the Site 465 report (this volume).

The MARs of the eolian component of the PlioPleistocene sediments at Site 465 are artificially low $\left(0.002\right.$ to $\left.0.009 \mathrm{~g} / \mathrm{cm}^{2} / 10^{3} \mathrm{yr}\right)$, roughly two orders of magnitude lower than values for sediments of the same age at other Hess Rise sites (Table 2). The percentage of the eolian component at Site $465(2-8 \%)$ is, however, similar to that at Site $466(2-18 \%)$ (Tables 2 and 3 ). Near the Cretaceous/Tertiary boundary, the MAR of the eolian component is also very low, averaging 0.016 and ranging up to $0.057 \mathrm{~g} / \mathrm{cm}^{2} / 10^{3} \mathrm{yr}$.

Samples from the organic-matter rich section of the Cenomanian to Albian laminated limestone show moderately high values for the accumulation of nonauthigenic, inorganic, crystalline components, but some of these values may be in error. We were unable to remove all the organic material from our samples from these intervals, although treatment with $\mathrm{H}_{2} \mathrm{O}_{2}$ seemed to remove most of it. Furthermore, the laminated limestones apparently were emplaced by down-slope transport from Mellish Bank (Site 465 report, this volume), so the inorganic MARs from this unit may represent

Table 1. Data generated for the calculation of MAR values for the non-authigenic, inorganic, crystalline, eolian (NICE) component of sediments recovered at DSDP Site 464.

\begin{tabular}{ccccccccc}
\hline $\begin{array}{c}\text { Sample } \\
\text { (interval in cm) }\end{array}$ & $\begin{array}{c}\text { Sub-bottom } \\
\text { Depth } \\
(\mathrm{m})\end{array}$ & $\begin{array}{c}\text { Age } \\
(\mathrm{m} . \mathrm{y})\end{array}$ & $\begin{array}{c}\text { Porosity } \\
(\%)\end{array}$ & $\begin{array}{c}\text { Dry-Bulk } \\
\text { Density } \\
\left(\mathrm{g} / \mathrm{cm}^{3}\right)\end{array}$ & $\begin{array}{c}\text { LSR } \\
\left(\mathrm{cm} / 10^{3} \mathrm{yr}\right)\end{array}$ & $\begin{array}{c}\text { MAR } \\
\left(\mathrm{g} / \mathrm{cm}^{2} / 10^{3} \mathrm{yr}\right)\end{array}$ & $\begin{array}{c}\text { NICE } \\
(\%)\end{array}$ & $\begin{array}{c}\text { NICE MAR } \\
\left(\mathrm{g} / \mathrm{cm}^{2} / 10^{3} \mathrm{yr}\right)\end{array}$ \\
\hline $464-2-2,40-44$ & 5.41 & 2.42 & 83.5 & 0.44 & 1.13 & 0.497 & 61.48 & 0.305 \\
$2-5,41-45$ & 9.92 & 2.82 & 79.5 & 0.54 & 1.13 & 0.610 & 33.07 & 0.202 \\
$3-2,80-84$ & 15.31 & 3.29 & 85.9 & 0.37 & 1.13 & 0.418 & 49.65 & 0.208 \\
$3-5,80-84$ & 19.81 & 3.69 & 82.8 & 0.46 & 1.13 & 0.520 & 59.58 & 0.310 \\
$4-2,110-114$ & 25.11 & 4.16 & 82.6 & 0.46 & 1.13 & 0.520 & 66.33 & 0.345 \\
$4-4,120-122$ & 28.21 & 4.43 & 83.6 & 0.43 & 1.13 & 0.486 & 47.52 & 0.231 \\
$5-2,134-138$ & 34.85 & 5.02 & 82.9 & 0.45 & 1.13 & 0.508 & 73.27 & 0.372 \\
$5-4,134-138$ & 37.85 & - & 68.4 & 0.84 & - & - & 69.21 & - \\
$6-2,22-24$ & 43.23 & - & 69.9 & 0.80 & - & - & 66.21 & - \\
$6-4,22-24$ & 46.23 & - & 70.5 & 0.78 & - & - & 73.18 & - \\
$7-2,130-132$ & 53.81 & - & 79.0 & 0.55 & - & - & 49.59 & - \\
$7-5,130-132$ & 58.31 & - & 81.7 & 0.48 & - & - & 58.57 & - \\
$8-2,68-70$ & 62.69 & - & 79.5 & 0.54 & - & - & 64.68 & - \\
$9-1,82-84$ & 70.83 & - & 81.6 & 0.49 & - & - & 70.36 & - \\
$9-6,82-84$ & 78.33 & - & 80.0 & 0.52 & - & - & 55.61 \\
$10-1,50-52$ & 80.01 & - & 81.4 & 0.49 & - & - & 60.73 \\
$10-3,50-52$ & 83.01 & - & 74.1 & 0.69 & - & - & - \\
\hline
\end{tabular}

Note: Rates for sediments below Core 5, Section 2 (the brown clays) are uncertain. 


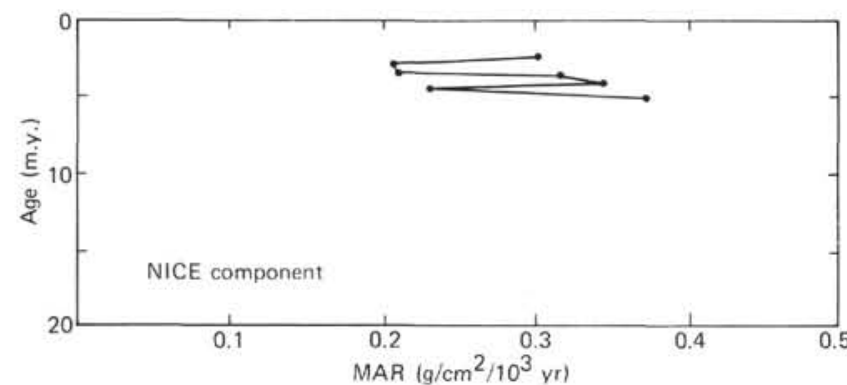

Figure 3. Mass-accumulation rates of the non-authigenic, inorganic, crystalline, eolian (NICE) component of deep-sea sediments from DSDP Site 464. See text.

more than just eolian materials. Data from the lower part of the laminated limestone of Unit II are not complicated by organic carbon and show high MARs for the inorganic component, averaging about $0.6 \mathrm{~g} / \mathrm{cm}^{2} / 10^{3}$ $\mathrm{yr}$, and reaching a maximum of $1.14 \mathrm{~g} / \mathrm{cm}^{2} / 10^{3} \mathrm{yr}$ just above the basal trachyte (Table 2). These values are similar to or somewhat higher than those from midCretaceous sediments at Site 463 and elsewhere in the Pacific (Rea and Janecek, this volume.)

\section{Site 466}

At Site 466, the most-complete Plio-Pleistocene section of the three Leg 62 drill sites on Hess Rise was cored. Lower in the section, we were able to analyze a few samples from Eocene, Campanian, and Albian materials. Linear sedimentation rates are from the 466 Site report (this volume).

The upper Cenozoic section (Table 3; Figs. 5 and 6) shows generally high, although fluctuating values for sediments about 3.8 to $5.8 \mathrm{~m}$.y. old. The average MAR for the eolian component during this interval is 0.22 $\mathrm{g} / \mathrm{cm}^{2} / 10^{3} \mathrm{yr}$. The mid-Pliocene to recent average MAR is $0.095 \mathrm{~g} / \mathrm{cm}^{2} / 10^{3} \mathrm{yr}$ and shows relative peaks at 1.4 and $0.2 \mathrm{~m} . \mathrm{y}$. The (reworked) Eocene materials in Cores 8 through 10 display a low eolian component, averaging

Table 2. Data generated for the calculation of MAR values for the non-authigenic, inorganic, crystalline, eolian (NICE) component of sediments recovered at DSDP Site 465 .

\begin{tabular}{|c|c|c|c|c|c|c|c|c|c|c|}
\hline $\begin{array}{c}\text { Sample } \\
\text { (interval in } \mathrm{cm} \text { ) }\end{array}$ & $\begin{array}{l}\text { Sub-bottom } \\
\text { Depth } \\
\text { (m) }\end{array}$ & $\begin{array}{c}\text { Age } \\
\text { (m.y.) }\end{array}$ & $\begin{array}{l}\text { Porosity } \\
(\%)\end{array}$ & $\begin{array}{l}\text { Dry-Bulk } \\
\text { Density } \\
\left(\mathrm{g} / \mathrm{cm}^{3}\right)\end{array}$ & $\begin{array}{c}\text { LSR } \\
\left(\mathrm{cm} / 10^{3} \mathrm{yr}\right)\end{array}$ & $\begin{array}{c}\text { MAR } \\
\left(\mathrm{g} / \mathrm{cm}^{2} / 10^{3} \mathrm{yr}\right)\end{array}$ & $\begin{array}{l}\text { NICE } \\
(\%)\end{array}$ & $\begin{array}{c}\text { NICE } \\
\text { After Removal of } \\
\text { Organic Matter } \\
(\%)\end{array}$ & $\begin{array}{l}\text { NICE MAR } \\
\left(\mathrm{g} / \mathrm{cm}^{2} / 10^{3} \mathrm{yr}\right)\end{array}$ & $\begin{array}{c}\text { NICE MAR } \\
\text { After Removal of } \\
\text { Organic Matter } \\
\left(\mathrm{g} / \mathrm{cm}^{2} / 10^{3} \mathrm{yr}\right)\end{array}$ \\
\hline $465-1-1,76-78$ & 0.77 & 1.0 & 54.8 & 1.20 & 0.10 & 0.120 & 7.69 & - & 0.009 & - \\
\hline $2-2,64-66$ & 3.15 & 2.5 & 62.4 & 0.99 & 0.10 & 0.099 & 3.60 & - & 0.004 & - \\
\hline $2-5,64-66$ & 7.65 & 4.0 & 59.7 & 1.06 & 0.10 & 0.106 & 2.109 & - & 0.002 & - \\
\hline $3-1,60-62$ & 11.11 & 55 & 59.7 & 1.07 & 0.80 & 0.856 & 2.88 & - & 0.025 & - \\
\hline $3-3,60-62$ & 14.11 & 56 & 58.4 & 1.10 & 0.80 & 0.880 & 0.28 & - & 0.002 & - \\
\hline $4-2,48-50$ & 21.99 & 57 & 55.5 & 1.18 & 0.80 & 0.944 & 0.31 & - & 0.003 & - \\
\hline $4-4,48-50$ & 24.99 & 57 & 53.8 & 1.22 & 0.80 & 0.976 & 1.39 & - & 0.014 & - \\
\hline $5-2,95-97$ & 31.96 & 57 & 52.0 & 1.27 & 0.80 & 1.016 & 0.43 & - & 0.004 & - \\
\hline $5-5,95-97$ & 36.46 & 58 & 50.6 & 1.31 & 0.80 & 1.048 & 0.58 & - & 0.006 & - \\
\hline $6-2,28-30$ & 40.79 & 58 & 49.7 & 1.33 & 0.80 & 1.064 & 0.707 & - & 0.007 & - \\
\hline $6-4,28-30$ & 43.79 & 59 & 55.3 & 1.18 & 0.80 & 0.994 & 1.32 & - & 0.013 & - \\
\hline $10-2,30-32$ & 78.81 & 65 & 57.3 & 1.13 & 0.70 & 0.791 & 0.50 & - & 0.004 & - \\
\hline $10-5,30-32$ & 83.31 & 66 & 56.3 & 1.16 & 0.70 & 0.812 & 0.22 & - & 0.002 & - \\
\hline $465 \mathrm{~A}-1-1,132-134$ & 40.33 & 58 & 52.4 & 1.26 & 0.30 & 0.378 & 2.21 & - & 0.008 & - \\
\hline $3-2,18-20$ & 59.69 & 64.5 & 60.9 & 1.03 & 0.30 & 0.309 & 0.89 & - & 0.003 & - \\
\hline $3-5,18-20$ & 64.69 & 66 & 56.5 & 1.15 & 0.80 & 0.920 & 0.59 & - & 0.005 & - \\
\hline $9-2,47-49$ & 116.98 & 69 & 70.2 & 0.79 & 4.20 & 3.318 & 0.65 & - & 0.020 & - \\
\hline $9-5,50-52$ & 121.51 & 69 & 56.6 & 1.10 & 4.20 & 4.620 & 0.95 & - & 0.044 & - \\
\hline $10-2,87-91$ & 126.88 & 69 & $54.8^{\mathrm{a}}$ & $1.20^{\mathrm{a}}$ & 4.20 & 5.040 & 0.63 & - & $0.032^{\mathrm{a}}$ & - \\
\hline $11-2,100-104$ & 136.51 & 69 & $52.2^{\mathrm{a}}$ & $1.26^{\mathrm{a}}$ & 4.20 & 5.292 & 0.12 & - & $0.006^{\mathrm{a}}$ & - \\
\hline $11-4,70-74$ & 139.21 & 69 & 55.0 & 1.19 & 4.20 & 4.998 & 0.43 & - & 0.022 & - \\
\hline $12-2,25-29$ & 145.26 & 70 & 55.5 & 1.17 & 4.20 & 4.914 & 0.23 & - & 0.011 & - \\
\hline $15-2,10-12$ & 173.61 & 70 & 55.3 & 1.18 & 4.20 & 4.956 & 1.17 & - & 0.057 & - \\
\hline $15-4,10-12$ & 176.61 & 70 & 53.7 & 1.23 & 4.20 & 5.166 & 0.41 & - & 0.021 & - \\
\hline $16-2,10-12$ & 183.11 & 70 & 54.9 & 1.20 & 4.20 & 5.040 & 0.35 & - & 0.017 & - \\
\hline $16-4,10-12$ & 186.11 & 70 & 53.0 & 1.24 & 4.20 & 5.208 & 0.62 & - & 0.032 & - \\
\hline $17-2,13-15$ & 192.64 & 70 & $47.9^{\mathrm{a}}$ & $1.38^{\mathrm{a}}$ & 4.20 & 5.796 & 0.51 & - & $0.029^{\mathrm{a}}$ & - \\
\hline $18-1,125-127$ & 201.76 & 70 & $57.2^{\mathrm{a}}$ & $1.13^{\mathrm{a}}$ & 4.20 & 4.746 & 0.57 & - & $0.027^{\mathrm{a}}$ & - \\
\hline $19-2,112-114$ & 212.63 & 70 & $52.8^{\mathrm{a}}$ & $1.25^{\mathrm{a}}$ & 4.20 & 5.250 & 0.33 & - & $0.017^{a}$ & - \\
\hline $20-2,15-17$ & 221.16 & 70 & 54.8 & 1.20 & 4.20 & 5.040 & 0.36 & - & 0.018 & - \\
\hline $21-4,4-6$ & 33.55 & 80 & 54.9 & 1.19 & 0.70 & 0.833 & 1.01 & - & 0.008 & - \\
\hline $26-1,65-66$ & 277.16 & 98.5 & 14.6 & 2.26 & 4.50 & 10.170 & $2.84^{\mathrm{b}}$ & 0.753 & $0.289^{\mathrm{b}}$ & 0.076 \\
\hline $27-1,78-79$ & 286.79 & 99.5 & 13.7 & 2.29 & 4.50 & 10.305 & $2.64^{\mathrm{b}}$ & - & $0.272^{b}$ & - \\
\hline $28-1,19-20$ & 29.70 & 100 & 13.9 & 2.28 & 4.50 & 10.260 & $2.10^{\mathrm{b}}$ & - & $0.215^{b}$ & - \\
\hline $29-1,26-27$ & 305.27 & 100 & 11.4 & 2.35 & 4.50 & 10.575 & $14.90^{\mathrm{b}}$ & - & $1.576^{\mathrm{b}}$ & - \\
\hline $30-1,36-38$ & 314.87 & 100 & 19.4 & 2.14 & 4.50 & 9.630 & $2.39^{\mathrm{b}}$ & - & $0.231^{\mathrm{b}}$ & - \\
\hline $31-1,5-7$ & 324.06 & 100 & 50.8 & 1.30 & 4.50 & 5.850 & $6.63^{\mathrm{b}}$ & - & $0.388^{b}$ & - \\
\hline $32-1,59-60$ & 336.10 & 100 & 30.9 & 1.83 & 4.50 & 8.235 & $2.20^{\mathrm{b}}$ & 0.333 & $0.182^{\mathrm{b}}$ & 0.027 \\
\hline $33-1,94-95$ & 343.95 & 100 & 30.7 & 1.84 & 4.50 & 8.280 & $2.19^{\mathrm{b}}$ & 0.026 & $0.181^{b}$ & 0.0021 \\
\hline $34-1,94-95$ & 353.45 & 101 & 23.4 & 2.03 & 4.50 & 9.135 & $2.18^{\mathrm{b}}$ & - & $0.199^{b}$ & - \\
\hline $36-2,90-91$ & 373.91 & 101 & 42.4 & 1.53 & 4.50 & 6.885 & 7.75 & - & 0.534 & - \\
\hline $37-2,77-78$ & 383.28 & 101 & $41.9^{\mathrm{a}}$ & $1.54^{\mathrm{a}}$ & 4.50 & 6.930 & 10.98 & - & $0.761^{\mathrm{a}}$ & - \\
\hline $38-1,75-76$ & 391.26 & 101 & 40.1 & 1.57 & 4.50 & 7.065 & 6.47 & - & 0.457 & - \\
\hline $39-1,115-116$ & 401.16 & 102 & 38.5 & 1.63 & 4.50 & 7.335 & 6.63 & - & 0.486 & - \\
\hline $40-1,45-46$ & 409.96 & 102 & 35.05 & 1.72 & 4.50 & 7.740 & 14.69 & - & 1.137 & - \\
\hline
\end{tabular}

a Samples that had undergone some drying (artifically lowered porosity values, and therefore larger MARs).

b Samples with a high organic-matter content (artificially high NICE and NICE MAR values). 


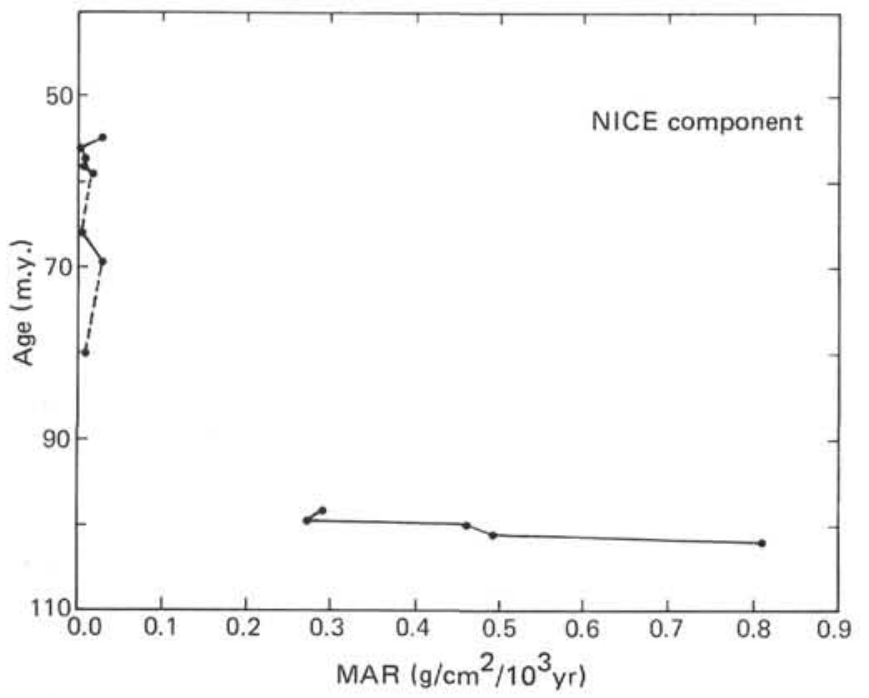

Figure 4. Mass-accumulation rates of the non-authigenic, inorganic, crystalline, eolian (NICE) component of deep-sea sediments from DSDP Site 465. See text.

about $0.04 \mathrm{~g} / \mathrm{cm}^{2} / 10^{3} \mathrm{yr}$. A single high value $(0.43$ $\mathrm{g} / \mathrm{cm}^{2} / 10^{3} \mathrm{yr}$ ) was obtained for upper Campanian sediments where the alkalic basalt pebbles were found (Core 12; Site 466 report, this volume). Upper Albian sediments, probably emplaced by turbidity currents, have a moderate to high MAR for the non-authigenic, inorganic, crystalline component, averaging 0.36 $\mathrm{g} / \mathrm{cm}^{2} / 10^{3} \mathrm{yr}$. These higher values are similar to Albian inorganic MARs from Site 463 (Rea and Janecek, this volume), and somewhat lower than those rates calculated for Site 465 .

\section{DISCUSSION}

The record of accumulation of eolian material on Hess Rise is far from complete, a result of large gaps in the depositional record and of very low recovery in the cherty Cretaceous sequences. Enough data do exist, however, to provide some insight into eolian MARs during Pleistocene to Pliocene time, Paleocene through Campanian time, and early Cenomanian to late Albian time.

Accumulation rates of the non-authigenic, inorganic, crystalline component of the deep-sea sediments on Hess Rise were moderately high during the late Cenozoic. At Sites 464 and 466, the MARs of this component are about 0.3 and $0.2 \mathrm{~g} / \mathrm{cm}^{2} / 10^{3} \mathrm{yr}$, respectively. The eolian MARs at Site 465 are unusually low, because of inaccuracies in dating the apparently incomplete PlioPleistocene section there (Site 465 report, this volume).

Details of the Plio-Pleistocene accumulation rates for the eolian component are shown in Figure 6. Data from Site 464, on northern Hess Rise, show fluctuating

Table 3. Data generated for the calculation of MAR values for the non-authigenic, inorganic, crystalline, eolian (NICE) component of sediments recovered at DSDP Site 466.

\begin{tabular}{|c|c|c|c|c|c|c|c|c|}
\hline $\begin{array}{c}\text { Sample } \\
\text { (interval in } \mathrm{cm} \text { ) }\end{array}$ & $\begin{array}{l}\text { Sub-bottom } \\
\text { Depth } \\
\text { (m) }\end{array}$ & $\begin{array}{c}\text { Age } \\
(\mathrm{m}, \mathrm{y} .)\end{array}$ & $\begin{array}{l}\text { Porosity } \\
(\%)\end{array}$ & $\begin{array}{l}\text { Dry-Bulk } \\
\text { Density } \\
\left(\mathrm{g} / \mathrm{cm}^{3}\right)\end{array}$ & $\begin{array}{c}\text { LSR } \\
\left(\mathrm{cm} / 10^{3} \mathrm{yr}\right)\end{array}$ & $\begin{array}{c}\text { MAR } \\
\left(\mathrm{g} / \mathrm{cm}^{2} / 10^{3} \mathrm{yr}\right)\end{array}$ & $\begin{array}{l}\text { NICE } \\
(\%)\end{array}$ & $\begin{array}{l}\text { NICE MAR } \\
\left(\mathrm{g} / \mathrm{cm}^{2} / 10^{3} \mathrm{yr}\right)\end{array}$ \\
\hline $466-1-1,31-34$ & 0.32 & - & 62.8 & 0.99 & 1.20 & 1.184 & 6.82 & 0.081 \\
\hline $1-2,110-112$ & 2.61 & 0.3 & 63.7 & 0.96 & 1.20 & 1.152 & 18.07 & 0.208 \\
\hline $1-4,40-43$ & 4.91 & 0.6 & 59.6 & 1.07 & 1.20 & 1.285 & 5.73 & 0.074 \\
\hline $1-5,110-112$ & 7.11 & 0.9 & 62.8 & 0.99 & 1.20 & 1.188 & 5.62 & 0.067 \\
\hline $2-1,20-23$ & 8.21 & 1.0 & 61.6 & 1.02 & 1.20 & 1.220 & 6.49 & 0.079 \\
\hline $2-2,65-67$ & 10.16 & 1.0 & 64.4 & 0.94 & 1.20 & 0.941 & 6.23 & 0.059 \\
\hline $2-4,63-65$ & 13.14 & 1.4 & 61.7 & 1.01 & 1.20 & 1.217 & 8.15 & 0.099 \\
\hline $2-6,65-67$ & 16.16 & 1.7 & 63.5 & 0.97 & 1.20 & 1.164 & 11.67 & 0.136 \\
\hline $3-2,67-68$ & 19.68 & 2.0 & 62.1 & 1.00 & 1.20 & 1.200 & 9.53 & 0.114 \\
\hline $3-3,67-68$ & 21.18 & 3.0 & 60.9 & 1.04 & 1.20 & 1.248 & 6.07 & 0.076 \\
\hline $3-5,99-101$ & 24.5 & 3.2 & 58.9 & 1.09 & 1.20 & 1.307 & 8.16 & 0.107 \\
\hline $4-1,10-13$ & 27.11 & 3.3 & 62.3 & 1.00 & 1.20 & 1.199 & 2.29 & 0.027 \\
\hline $4-2,58-60$ & 29.09 & 3.4 & 57.5 & 1.12 & 1.20 & 1.344 & 7.89 & 0.106 \\
\hline $5-1,20-23$ & 36.71 & 3.8 & 58.4 & 1.10 & 4.10 & 4.518 & 3.64 & 0.164 \\
\hline $5-2,65-67$ & 38.66 & 3.9 & 60.4 & 1.05 & 4.10 & 4.305 & 4.72 & 0.203 \\
\hline $5-4,65-67$ & 41.66 & 4.0 & 59.4 & 1.07 & 4.10 & 4.387 & 4.03 & 0.177 \\
\hline $6-1,20-23$ & 46.21 & 4.3 & 59.5 & 1.07 & 4.10 & 4.403 & 5.22 & 0.229 \\
\hline $6-2,130-132$ & 48.81 & 4.5 & 61.5 & 1.02 & 4.10 & 4.182 & 4.38 & 0.183 \\
\hline $6-4,130-132$ & 51.81 & 4.7 & 59.1 & 1.08 & 4.10 & 4.444 & 4.23 & 0.188 \\
\hline $6-6,130-132$ & 63.81 & 4.9 & 63.1 & 0.98 & 4.10 & 4.018 & 8.52 & 0.342 \\
\hline $7-1,50-53$ & 56.01 & 5.0 & 61.0 & 1.03 & 4.10 & 4.232 & 4.98 & 0.211 \\
\hline $7-2,50-52$ & 57.51 & 5.0 & 67.0 & 0.87 & 4.10 & 3.567 & 4.54 & 0.162 \\
\hline $7-3,30-33$ & 58.81 & 5.0 & 64.3 & 0.95 & 4.10 & 3.879 & 4.84 & 0.188 \\
\hline $7-5,50-52$ & 62.01 & 5.0 & 61.0 & 1.03 & 4.10 & 4.223 & 9.52 & 0.402 \\
\hline $8-2,62-64$ & 67.13 & 37 & 54.2 & 1.21 & 0.20 & 0.242 & 5.70 & 0.014 \\
\hline $8-5,62-64$ & 71.63 & 39.5 & 44.9 & 1.46 & 0.20 & 0.292 & 6.04 & 0.018 \\
\hline $9-2,65-67$ & 76.66 & 40 & 52.4 & 1.26 & 0.20 & 0.252 & 2.83 & 0.007 \\
\hline $9-4,65-67$ & 79.66 & 46.5 & 55.9 & 1.17 & 0.20 & 0.234 & 5.14 & 0.012 \\
\hline $10, \mathrm{CC}$ & 84.1 & 49 & 46.6 & 1.41 & 0.20 & 0.228 & 23.10 & 0.053 \\
\hline $12-1,82-84$ & 94.33 & 70 & 55.6 & 1.18 & 4.30 & 5.074 & 8.48 & 0.430 \\
\hline $13-2,45-47$ & 96.96 & 70 & 62.3 & 1.00 & 4.30 & 4.300 & 1.10 & 0.047 \\
\hline $13-5,45-47$ & 99.96 & 70.2 & 55.8 & 1.17 & 4.30 & 5.031 & 0.32 & 0.016 \\
\hline $15-2,82-84$ & 124.33 & 70.9 & 56.9 & 1.14 & 4.30 & 4.902 & 0.64 & 0.031 \\
\hline $15-4,82-84$ & 127.33 & 71 & 58.5 & 1.10 & 4.30 & 4.730 & 1.13 & 0.053 \\
\hline $16-2,64-66$ & 133.65 & 81 & 52.6 & 1.26 & 2.80 & 3.528 & 1.33 & 0.047 \\
\hline $17-1,94-96$ & 141.95 & 81 & 58.0 & 1.11 & 2.80 & 3.108 & 1.68 & 0.052 \\
\hline $29-1,20-22$ & 255.21 & 100 & 46.5 & 1.42 & 2.80 & 3.976 & 7.91 & 0.314 \\
\hline $30-1,50-52$ & 265.01 & 100.5 & 45.2 & 1.45 & 2.80 & 4.060 & 8.74 & 0.355 \\
\hline $34-1,51-52$ & 293.52 & 101.5 & 34.1 & 1.75 & 2.80 & 4.900 & 7.82 & 0.383 \\
\hline $35-1,62-63$ & 303.13 & 102 & 49.2 & 1.35 & 2.80 & 3.780 & 9.99 & 0.378 \\
\hline
\end{tabular}




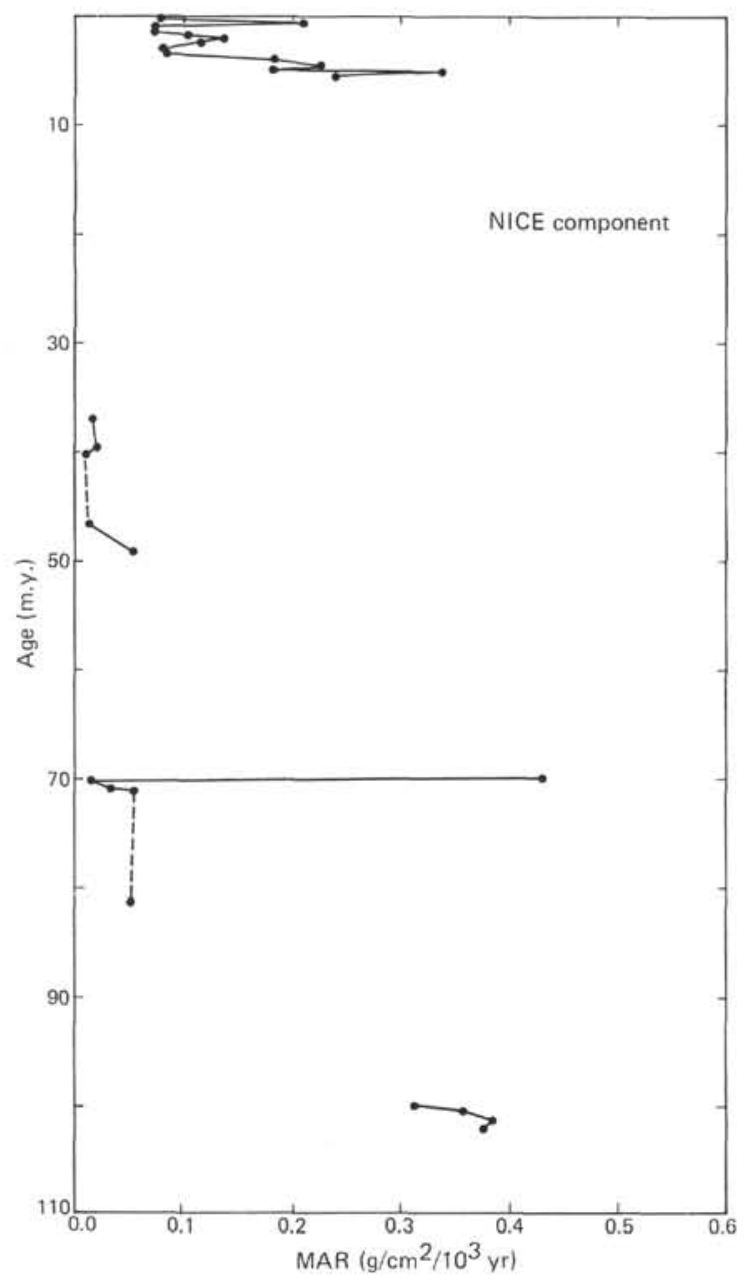

Figure 5. Mass-accumulation rates of the non-authigenic, inorganic, crystalline, eolian (NICE) component of deep-sea sediments from DSDP Site 466. See text.

values from Pliocene sediments about 2.4 to 5.0 m.y. old. The MAR pattern at Site 464 apparently contains no unique information; it broadly conforms to the general pattern of higher eolian MARs 4 to $5 \mathrm{~m} . \mathrm{y}$. ago, and relatively lower values about $3 \mathrm{~m} . \mathrm{y}$. ago, that can be seen in the data from Site 466 (Fig. 6) and from Site 463 (Rea and Janecek, this volume).

Site 466 contains the most complete record of the three Hess Rise Plio-Pleistocene sections drilled during Leg 62 . We sampled this section in greater detail than the others, hoping to learn more about the periodic volcanism and the climatic changes of the late Cenozoic. Smear slides of these sediments show as much as $5 \%$ volcanic glass, and traces of clay minerals and zeolites. Between about 3.8 and 5.4 m.y. ago, the accumulation of eolian material averaged about $0.22 \mathrm{~g} / \mathrm{m}^{2} / 10^{3} \mathrm{yr}$. More recently at Site 466 , the average has been about $0.1 \mathrm{~g} / \mathrm{m}^{2} / 10^{3} \mathrm{yr}$ (Table 3; Fig. 6). The accumulation maximum of the eolian component between roughly 4 and $5 \mathrm{~m} . \mathrm{y}$. ago also occurs at Site 463 (Rea and Janecek, this volume), and ash layers of this age are common throughout the world's oceans (Kennett and Thunell, 1977). Both circum-Pacific (Kennett et al., 1977) and mid-plate (Rea and Scheidegger, 1979) volcanic activity were relatively high during the early Pliocene, and this

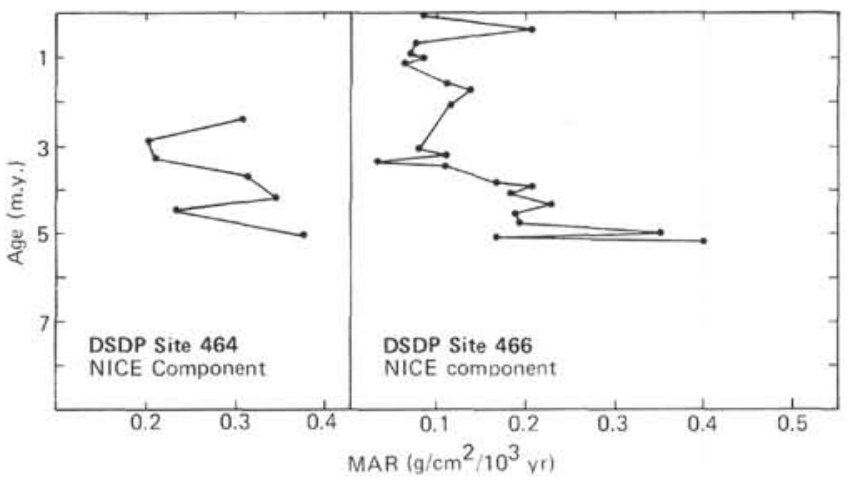

Figure 6. Details of Plio-Pleistocene eolian MARs from DSDP Sites 464 and 466 . See text.

appears to be the direct cause of the high MARs 4 to 5 m.y. ago at Site 466 .

Between 3.8 m.y. ago and the present, the eolian MAR at Site 466 has been about $0.1 \mathrm{~g} / \mathrm{cm}^{2} / 10^{3} \mathrm{yr}$ and displays relative lows at 3 and $1 \mathrm{~m} . \mathrm{y}$. ago, with higher values 1.5 to 2 and about 0.2 m.y. ago (Fig. 6). This pattern is different from that calculated for the other two locations in the North Pacific where appropriate data exist. About $2200 \mathrm{~km}$ east of Site 466, and at a similar latitude, a large-diameter piston core of red clay (LL44GPC $3 ; 30.3^{\circ} \mathrm{N}, 157.8^{\circ} \mathrm{W}$ ) reveals a similar MAR for the eolian component, but shows a pattern of roughly fivefold increase in that MAR value since the late Miocene (Leinen et al., 1979). At Site 463, $1400 \mathrm{~km}$ south of Site 466 , there has also been a five-fold increase in the eolian MAR since late Miocene time, although the actual values of inorganic MAR are almost an order of magnitude less than at Site 466 (Rea and Janecek, this volume). The absence of the upper Miocene at Site 466 precludes an evaluation of this anticipated relative increase in eolian MARs.

During the Maastrichtian to early Campanian, the mass-accumulation rate of non-authigenic, inorganic, crystalline material on Hess Rise was very low; MARs averaged less than 0.02 and about $0.04 \mathrm{~g} / \mathrm{cm}^{2} / 10^{3} \mathrm{yr}$ for Sites 465 and 466, respectively. Smear-slide and carbonate-percent data from Site 310 , on central Hess Rise (Larson, Moberly, et al., 1975), also suggest a very low accumulation rate of eolian material during this same period.

This low MAR for the eolian component on Hess Rise is in marked contrast to MAR data for the same time from drill sites to the south. At Site 463, in the western Mid-Pacific Mountains, the eolian MAR for lower Maestrichtian materials is about $0.2 \mathrm{~g} / \mathrm{cm}^{2} / 10^{3} \mathrm{yr}$ (Rea and Janecek, this volume). In the Nauru Basin, at Site 462 , it is even higher, averaging $0.6 \mathrm{~g} / \mathrm{cm}^{2} / 10^{3} \mathrm{yr}$ for the interval 69 to 77 m.y. ago (Rea and Thiede, in press). Many other sites in the central Pacific contain more-qualitative data recording an increase in volcanic activity during Campanian to Maastrichtian time (Site 61, Leg 7, Winterer, Riedel, et al., 1971; Sites 196, 198, and 199, Leg 20, Heezen, MacGregor, et al., 1973; Site 313, Leg 32, Larson, Moberly, et al., 1975; Sites 315 and 316, Leg 33, Schlanger, Jackson, et al., 1976). There seems little doubt that many of the volcanic 
edifices now lying in the equatorial and subtropical Pacific were created during this period of eruptive activity ( $c f$. Winterer, 1976).

Cores from Sites 465 and 466 show large MARs of the non-authigenic, inorganic, crystalline component in the lower Cenomanian and upper Albian laminated limestone and chalk turbidites (Figs. 4 and 5). Some of the MAR values from the Cenomanian samples of Site 465 are large because of their high organic-matter content, but the underlying Albian materials are not organic-matter-rich and display an average inorganic MAR of about $0.6 \mathrm{~g} / \mathrm{cm}^{2} / 10^{3} \mathrm{yr}$ (Table 2). At Site 466 , this value is $0.35 \mathrm{~g} / \mathrm{cm}^{2} / 10^{3} \mathrm{yr}$ (Table 3).

Hess Rise basement is Albian, as indicated by drilling results at Site 464 and 465 (Site 464 and Site 465 reports, this volume), so indications of Albian volcanism are to be expected. Mellish Bank, the shallowest, southern portion of Hess Rise is underlain by trachyte, a latestage differentiate of alkalic basalt, suggesting that volcanism may have continued in this region longer than it did farther north.

Mass-accumulation rates of this component averaged about $0.27 \mathrm{~g} / \mathrm{cm}^{2} / 10^{3} \mathrm{yr}$ at Site 463 in the Mid-Pacific Mountains (Rea and Janecek, this volume) during Albian time. Data from the Line Islands (Winterer, Ewing, et al., 1973) and the Nauru Basin (Rea and Thiede, in press) also reflect a moderate amount of midCretaceous volcanism. Albian to Cenomanian volcanism apparently was a common phenomenon in the central Pacific (Winterer, 1976).

\section{SUMMARY}

Samples from three drill sites on Hess Rise were treated chemically to remove the calcareous, opaline, and authigenic fractions of the sediment. The remaining fraction is presumed to represent the influx of eolian material to Hess Rise, except for the mid-Cretaceous limestone turbidites. Variations in the mass-accumulation rate of this component appear to reflect fluctuations in the influx of volcanogenic material, except perhaps during the late Cenozoic glacial ages. As a result of long hiatuses and very low recovery in some sections, only a discontinuous record of eolian influx can be constructed (Figs. 3, 4, and 5).

Ash layers just above the trachyte basement at Site 465 record the waning of Albian volcanism that constructed Hess Rise; MARs of the inorganic component for the late Albian and early Cenomanian may reflect bottom transport of material as well as eolian influx and are reasonably high $-0.6 \mathrm{~g} / \mathrm{cm}^{2} / 10^{3} \mathrm{yr}$ at Site 465 , and $0.35 \mathrm{~g} / \mathrm{cm}^{2} / 10^{3}$ yr at Site 466 , somewhat farther from the possible source region on Mellish Bank. This midCretaceous volcanism on Hess Rise appears to be part of an episode of volcanic activity that spanned much of the west-central Pacific.

Mass-accumulation rates of the eolian component were very low from the Paleocene through the Campanian, averaging roughly $0.03 \mathrm{~g} / \mathrm{cm}^{2} / 10^{3} \mathrm{yr}$ at Sites 465 and 466. Low MARs for the Paleocene volcanic component have been found in sediments at Site 462 in the Nauru Basin $\left(0.06 \mathrm{~g} / \mathrm{cm}^{2} / 10^{3} \mathrm{yr}\right.$; Rea and Thiede, in press), but many DSDP sites in the central Pacific record a significant influx of volcanic materials during Maastrichtian to Campanian time. None of the Hess Rise sites contain evidence for a late Cretaceous volcanic maximum, indicating that this event was confined to more southerly locations.

Pliocene and Pleistocene accumulation of eolian materials was moderately high at two of the three Hess Rise sites $\left(0.3\right.$ and $0.2 \mathrm{~g} / \mathrm{cm}^{2} / 10^{3} \mathrm{yr}$ at Sites 464 and 465 ), but only $0.005 \mathrm{~g} / \mathrm{cm}^{2} / 10^{3} \mathrm{yr}$ at Site 465 . The general pattern of Plio-Pleistocene eolian deposition on Hess Rise (Fig. 6) reveals a maximum MAR 4 to 5 m.y. ago, a low centered about 3 m.y. ago, and slightly higher but fluctuating values since then. The peak at 4 to $5 \mathrm{~m} . \mathrm{y}$. ago is thought to reflect the Pacific-wide volcanic maximum that occurred in early Pliocene time (Kennett et al., 1977; Rea and Scheidegger, 1979). Data from other locations in the Pacific show a several-fold increase in the eolian MAR during the last 2 or 3 m.y., associated with the onset of glaciation (Leinen et al., 1979; Rea and Janecek, this volume), but this MAR increase is not as clear-cut at Site 466. Perhaps other kinds of data from Site 466 , such as the grain size and mineralogy of the eolian component, would give more obvious information on this topic.

\section{ACKNOWLEDGMENTS}

We would like to thank Tom Janecek for assistance with the laboratory work. Margaret Leinen and Ted Moore reviewed the manuscript, and we appreciate their comments and suggestions.

Portions of the work presented here were supported by NSF Grant OCE-7825341.

CLIMAP Project Members, 1976. The surface of the ice-age earth. Science, 191:1131-1137.

Heezen, B. C., MacGregor, I. D., et al., 1973. Init. Repts. DSDP, 20: Washington (U.S. Govt. Printing Office).

Kennett, J. P., and Thunell, R. C., 1977. On explosive Cenozoic volcanism and climatic implications. Science, 196:1231-1234.

Kennett, J. P., McBirney, A. R., and Thunell, R. C., 1977. Episodes of Cenozoic volcanism in the circum-Pacific region. J. Volcanol. Geothermal Res., 2:145-163.

Larson, R. L., Moberly, R., et al., 1975. Init. Repts. DSDP, 32: Washington (U.S. Govt. Printing Office).

Leinen, M., Heath, G. R., and Rea, D. K., 1979. Paleo-eoliata: sedimentary indicators of Cenozoic atmospheric circulation in the Northern Hemisphere. Geol. Soc. Am. Abs. Prog., 11:465. (Abstract)

Rea, D. K., and Scheidegger, K. F., 1979. Eastern Pacific spreading rate fluctuation and its relation to Pacific area volcanic episodes. J. Volcanol. Geothermal Res., 5:135-148.

Rea, D. K., and Thiede, J., in press. Mesozoic and Cenozoic mass accumulation rates of the major sediment components in the Nauru Basin, western equatorial Pacific. In Larson, R. L., Schlanger, S.O., et al., Init. Repts. DSDP, 61: Washington (U.S. Govt. Printing Office).

Schlanger, S. O., Jackson, E. D., et al., 1976. Init. Repts. DSDP, 33: Washington (U.S. Govt. Printing Office).

van Andel, Tj. H., Heath, G. R., and Moore, T. C., Jr., 1975. Cenozoic History and Paleoceanography of the Central Equatorial Pacific Ocean: Geol. Soc. Am. Mem., 143.

Winterer, E. L., 1976. Anomalies in the tectonic evolution of the Pacific. In Sutton, G. H., Manghnani, M. H., and Moberly, R. (Eds.), The Geophysics of the Pacific Ocean Basin and its Margin: Washington (Am. Geophys. Union), pp. 269-278.

Winterer, E. L., Ewing, J. I., et al., 1973. Init. Repts. DSDP, 17: Washington (U.S. Govt. Printing Office).

Winterer, E. L., Riedel, W. R., et al., 1971. Init. Repts. DSDP, 7: Washington (U.S. Govt. Printing Office). 